\title{
Formation of aluminum nanodot array by combination of nanoindentation and anodic oxidation of aluminum
}

\author{
Shoso Shingubara *, Yusuhiko Murakami, Kazunori Morimoto, \\ Takayuki Takahagi
}

Graduate School of Advanced Sciences and Matters, Hiroshima University, 1-3-1 Kagamiyama, Higashi-Hiroshima 739-8530, Japan

\begin{abstract}
Porous alumina films formed by aluminum anodic oxidation have been intensively studied to use them as molds to form nanowires or dots. Recently we established the formation of porous alumina on solid substrates such as $\mathrm{Si}$, and found a formation of an ordered aluminum hexagonal dot array after finishing of anodic oxidation on $\mathrm{SiO}_{2}$ substrate. We investigated AFM nanoindentation to control the initial position of nanoholes during anodic oxidation, in order to realize nanoholes and dots with various sizes and densities. Arrays of nanoholes with a nearest neighbor distance from 50 to $120 \mathrm{~nm}$ were successfully formed. Al dot tetragonal and hexagonal arrays were formed after selective wet chemical etching of porous alumina film. Electron transport through Al nanodots array at low temperature exhibited a nonlinear characteristic with a suppression of current around zero bias, which strongly suggests existence of Coulomb blockade.
\end{abstract}

(c) 2003 Published by Elsevier Science B.V.

Keywords: Electrochemical methods; Electrical transport measurements; Aluminum; Aluminum oxide; Electrical transport (conductivity, resistivity, mobility, etc.); Self-assembly; Surface structure, morphology, roughness, and topography; Solid-liquid interfaces

\section{Introduction}

Porous alumina films formed by anodic oxidation of aluminum (Al) have been intensively studied for use as molds to form nanowires or dots by depositing various metals or semiconductors in them, since highly ordered nanohole arrays can be formed [1-12]. By the use of oxalic acid, sulfuric acid, or phosphoric acid, trigonal lattices of alu-

\footnotetext{
${ }^{*}$ Corresponding author. Address: Department of Electrical Engineering, Hiroshima University, 1-4-1 Kagamiyama, Higashi-Hiroshima 739-8527, Japan. Tel.: +81-824-24-7645; fax: +81-824-22-7195.

E-mail address: shingu@hiroshima-u.ac.jp (S. Shingubara).
}

mina nanoholes with distances of several tens of $\mathrm{nm}$ can be formed under the self-organization conditions of anodic oxidation. We recently succeeded in forming an $\mathrm{Al}$ dot hexagonal array on a $\mathrm{SiO}_{2} / \mathrm{Si}\left(\begin{array}{lll}1 & 0 & 0\end{array}\right)$ substrate through adequate conditions of anodic oxidation and the subsequent selective removal of porous alumina film [13-15]. Al dots measuring a few tens of $\mathrm{nm}$ were formed at an interface between the porous alumina film and the $\mathrm{SiO}_{2}$ film by a break-up of $\mathrm{Al}$ film due to completion of anodic oxidation. These Al dots are useful for application to quantum effect devices such as single electron memory nodes or quantum cellular automata devices based on tunneling phenomena within two-dimensional quantum dot 
arrays [16]. However, the size and geometrical arrangement of the ordered porous alumina nanohole array are restricted by the self-organization conditions of each acid species. For example, the nearest neighbor distances of nanoholes at the selforganization condition are 475, 90 and $60 \mathrm{~nm}$ for phosphoric acid (anodization voltage $V_{\mathrm{a}}=195 \mathrm{~V}$ ) [10], oxalic acid $\left(V_{\mathrm{a}}=40 \mathrm{~V}\right)[7,8]$, and sulfuric acid $\left(V_{\mathrm{a}}=28 \mathrm{~V}\right)$ [9], respectively. Furthermore, the arrangement of nanoholes is limited to trigonal lattices only. It is desirable to form an ordered nanohole array with a desired dimension and geometry, and Masuda et al. proposed pretexturing of aluminum surface using $\mathrm{SiC}$ molds that had lithographically delineated periodic convexes $[17,18]$.

In this study, at first, detail characterizations of $\mathrm{Al}$ dots formed on $\mathrm{SiO}_{2} / \mathrm{Si}$ substrate were made by atomic force microscopy (AFM) and transmission electron microscopy (TEM) observations. Then a control of a geometrical arrangement of a nanohole array as well as Al dots by the use of AFM nanoindentation were investigated. We focused on the effect of indentation interval on a regularity of nanohole array in this experiment. Finally, electrical conduction characteristics between $\mathrm{Al}$ dots were examined by attaching electrodes using focused ion beam induced tungsten deposition.

\section{Results and discussion}

In order to form porous alumina film on $\mathrm{SiO}_{2} / \mathrm{Si}$ substrate, we deposited pure aluminum $(99.999 \%)$ film by sputtering on the substrate. The essential point to get well-ordered nanohole array for sputtered Al film by anodic oxidation is to keep aluminum surface flat. The regularity of nanohole arrangement is delicately dependent on the aluminum surface roughness, and usually regularity is poor when the surface roughness is large. For this reason, electropolishing with a diluted perchloric acid is carried out in the most cases of anodic oxidation for bulk Al. In the present study using sputtered Al films, it is difficult to deposit thick Al film to undergo electropolishing, hence we optimized sputtering conditions to keep surface as flat as can. The typical sputtering rate of $\mathrm{Al}$ is about
$10-15 \mathrm{~nm} / \mathrm{min}$, and substrate was tightly attached to water-cooled copper block, and furthermore, sputtering was carried out by alternation of $1 \mathrm{~min}$ deposition and $1 \mathrm{~min}$ rest so as not to raise substrate temperature. By this method, aluminum film surface roughness could be controlled within $2 \mathrm{~nm}$ in root mean square roughness (RMS) even for Al films which thickness were larger than $10 \mu \mathrm{m}$.

When an anodic oxidation of $\mathrm{Al}$ film is carried out with the typical self-organization conditions of $40 \mathrm{~V}$ at $2{ }^{\circ} \mathrm{C}$ in $0.15 \mathrm{M}$ oxalic acid solution, wellordered nanohole array is obtained when $\mathrm{Al}$ film thickness is larger than $10 \mu \mathrm{m}$. By a so-called two step anodic oxidation in which a properly long first anodization and selective removal of porous alumina film were carried out, a well ordered nanohole array is formed in the second anodic oxidation [7]. Poly-crystalline domain structure of nanohole trigonal lattice is formed, and the domain size increases with an increase in the first step anodic oxidation time. It is not clear whether domain boundary corresponds to the crystalline grain boundary. The domain size might be limited by the grain size in the cases of self-organized formation of nanohole arrays.

When a long time anodic oxidation is carried out, most of the Al film is converted to porous alumina. We found a formation of $\mathrm{Al}$ dot at the interface between porous alumina and $\mathrm{SiO}_{2}$. Initial sputtered Al thickness was $20 \mu \mathrm{m}$, and anodic oxidation was carried out with $40 \mathrm{~V}$ by $0.15 \mathrm{M}$ oxalic acid. Cross-sectional views of $\mathrm{Al}$ dots are shown in Fig. 1. Scanning electron microscopy (SEM) micrographs of the nanohole bottoms shown in Fig. 1(a) and (b) were taken at different locations on the same sample formed by $10 \mathrm{~h}$ anodic oxidation. These micrograph was taken after cutting the $\mathrm{Si}$ substrate, and the $\mathrm{Al}$ cross-section was slightly elongated. In some areas, Al film was still continuous while periodic roughness reflecting hemispherical nanohole bottoms existed as Fig. 1(a). However, Al film broke-up to individual dots in other areas as seen in Fig. 1(b). A non-uniformity of the rate of anodic oxidation would be ascribed to distribution of electric field because electrode was contacted at the limited area of the Al surface. With an enough long anodic oxidation of $11 \mathrm{~h}$, the entire $\mathrm{Al}$ dots were converted to 
(a)

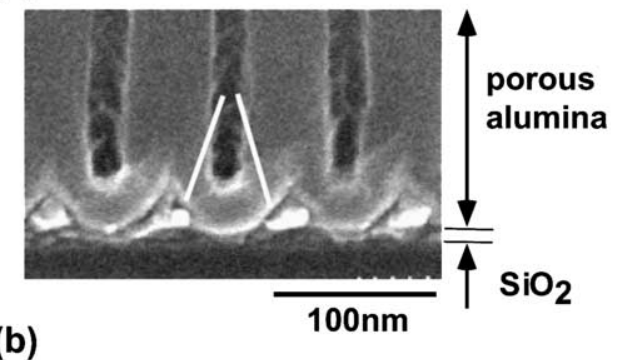

(b)

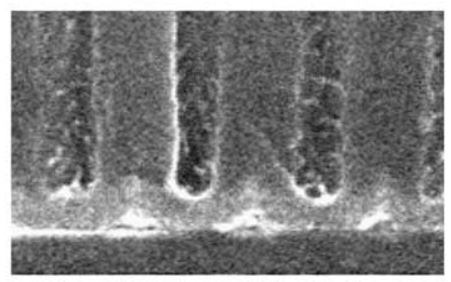

(c)

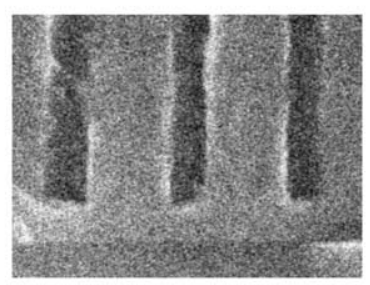

(d)

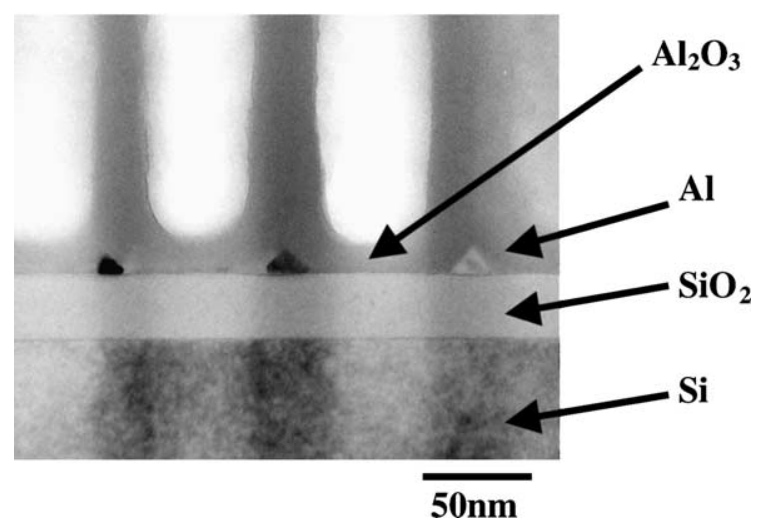

Fig. 1. Cross-sectional view of $\mathrm{Al}$ dots formed by anodic oxidation of sputtered $\mathrm{Al}$ film on $\mathrm{SiO}_{2}(50 \mathrm{~nm}) / \mathrm{Si}$ substrate. Initial sputtered Al thickness was $20 \mu \mathrm{m}$, and anodic oxidation was carried out with $40 \mathrm{~V}$ by $0.15 \mathrm{M}$ oxalic acid. (a-c) SEM crosssectional micrographs of the nanohole bottoms. (a and b) Observations at different locations on the same sample with $10 \mathrm{~h}$ anodic oxidation. (c) Sample with $11 \mathrm{~h}$ anodic oxidation. (d) Cross-sectional TEM micrograph of $\mathrm{Al}$ dots after $10.5 \mathrm{~h}$ anodic oxidation. alumina as shown in Fig. 1(c). Thus anodic oxidation gradually proceeds even after breaking-up of Al film to dots, and this phenomena was supposed to be carried out by ionic conduction within anodic alumina film. A cross-sectional TEM micrograph of Al dots (Fig. 1d) clearly showed crystalline phase of $\mathrm{Al}$ dot.

AFM observations of the remaining $\mathrm{Al}$ after selective wet chemical etching of porous alumina using a mixture of chromic acid and phosphoric acid are shown in Fig. 2. The observed sample was formed by $10.5 \mathrm{~h}$ and $40 \mathrm{~V}$ anodic oxidation by $0.15 \mathrm{M}$ oxalic acid with the initial $\mathrm{Al}$ thickness of $20 \mu \mathrm{m}$. It is clear that $\mathrm{Al}$ hexagonal nanodot array is formed on a flat surface of $\mathrm{SiO}_{2}$. The diameter and height of the $\mathrm{Al}$ dots are 40 and $15 \mathrm{~nm}$, respectively, and the nearest-neighbor distance of the $\mathrm{Al}$ dots is $65 \mathrm{~nm}$. The diameter of the hexagon is equal to the cell size of the nanoholes. This is one typical example of $\mathrm{Al}$ dot array, however, there is a serious problem in realizing large area $\mathrm{Al}$ dot array because non-uniformities existed in the dot size as well as in the inter-dot spacing. They

(a)

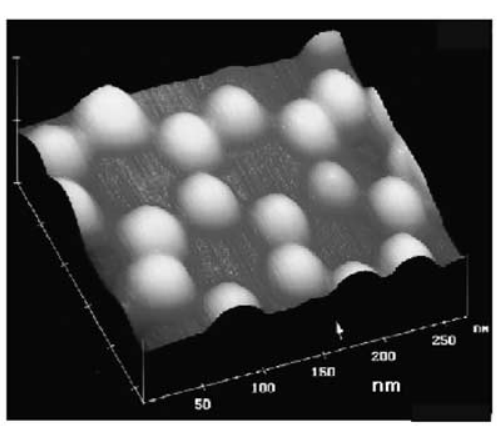

(b)

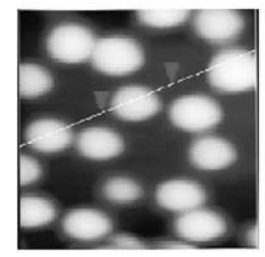

(c)

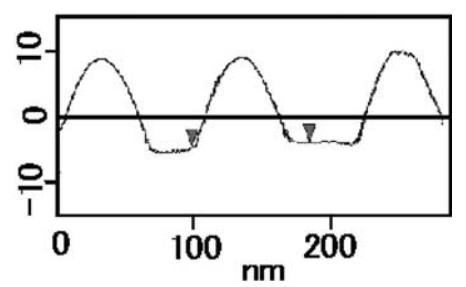

Fig. 2. AFM observations and its height analysis of Al hexagonal dot array on $\mathrm{SiO}_{2}$, that remained after selective wet chemical etching of porous alumina film. 
arose from non-uniformity of anodic oxidation speed with a very thick initial Al thickness of 20 $\mu \mathrm{m}$. If we use a thinner $\mathrm{Al}$ film with a shorter first step anodization time, regularity of nanohole array as well as Al dot array is poor.

Then, we tried to control the size and arrangement of a nanohole array, by making an array of concavities using AFM nanoindentation prior to $\mathrm{Al}$ anodic oxidation [15]. As a matter of fact, it turned out that the use of a thin $\mathrm{Al}$ film was essential in the control of nanoholes and Al dots by pretexturing techniques using AFM nanoindentation. The fabrication procedure of the nanohole and $\mathrm{Al}$ dot array is shown schematically in Fig. 3. We formed an array of concavities at a constant interval from 50 to $150 \mathrm{~nm}$ by an AFM nanoindention on the surface of a sputtered Al film with a thickness of $100 \mathrm{~nm}$ (Fig. 3a). Next, a nanohole array was formed by the anodic oxidation using $0.15 \mathrm{M}$ oxalic acid at a DC voltage of $30-40 \mathrm{~V}$ (Fig. 3b). Finally, the alumina film was selectively etched away. Al dots that existed at an interface between the porous alumina and $\mathrm{SiO}_{2}$ films then appeared on the surface (Fig. 3c). We used MMAFM of Beeco Corp. equipped with a diamond tip cantilever, which was specially designed for nanoindentation, with a curvature of $10 \mathrm{~nm}$.

At first we examined the effect of the indentation strength on the nanohole arrangement in the case of tetragonal arrays. Tetragonal hole arrays are known to be unstable when they are created by the periodic concavity formed by indentation using a $\mathrm{SiC}$ mold prior to anodic oxidation $[17,18]$. Tetragonal hole arrays tend to rearrange into trigonal ones during growth of nanoholes by anodic oxidation. If Al film thickness is too large, con- version from tetragonal array to trigonal array proceeds. We used anodic voltage between 30 and $40 \mathrm{~V}$; in these conditions, nanohole nearest neighbor distance is between 65 and $90 \mathrm{~nm}$. We observed tetragonal arrays were stable when the Al film thickness was smaller than $0.5 \mu \mathrm{m}$. In the following experiments, we used $\mathrm{Al}$ films with 0.1 $\mu \mathrm{m}$ thickness.

We examined a dependence of alumina nanohole arrangement on the indentation strength $F$. When the indentation strength $F$ was lower than $2.0 \times 10^{-5} \mathrm{~N}$, depths of concavities formed by indentation were equivalent or less to the average roughness of the $\mathrm{Al} \mathrm{film}$, and the porous alumina film surface morphology did not show any clear periodicity. In fact, the RMS of the $100 \mathrm{~nm}$ thick Al film was about $2.0 \mathrm{~nm}$, and the depth of concavity formed by AFM nanoindentation distributed between 1.0 and $2.5 \mathrm{~nm}$. When the indentation strength was increased to $4.0 \times 10^{-5} \mathrm{~N}$, the concavities depth formed by indentation distributed between 15 and $18 \mathrm{~nm}$, and a very clear periodic tetragonal array of nanoholes was formed after anodic oxidation. Scanning speed of nanoindentation is another important factor to form clearly separated nanoholes. When scanning speed was too high, concavities made by nanoindentation were connected each other and finally nanoholes formed by anodic oxidation were connected. We adopted a scanning speed slower than $10 \mathrm{dots} / \mathrm{s}$ for nanoindentation experiments with the indentation force of $4.2 \times 10^{-5} \mathrm{~N}$. This rate is relatively very slow, and it takes pretty long time when a large area is nanoindented.

Next we investigated the effect of an indentation interval $\left(d_{\text {int }}\right)$ on the spatial order of nanoholes
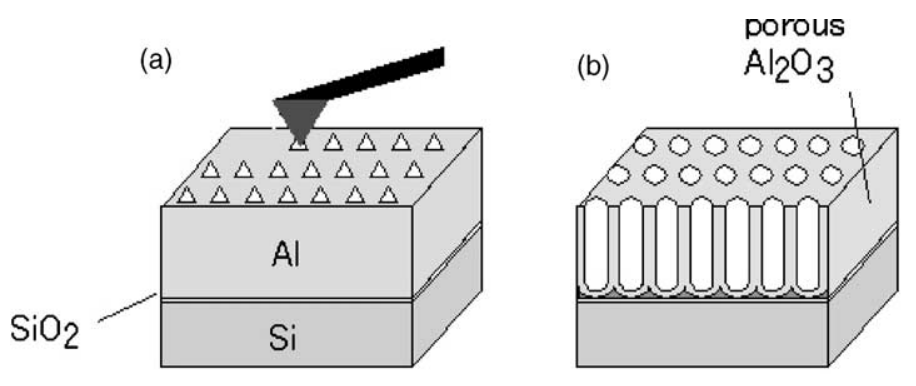

(c)

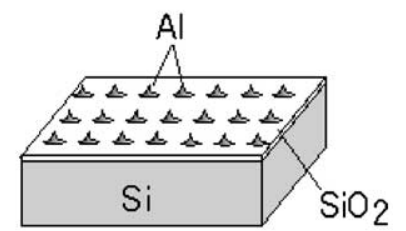

Fig. 3. Schematic diagram of $\mathrm{Al}$ nanodot array fabrication procedures. 
(a) $d_{\text {int }}=55 \mathrm{~nm}$

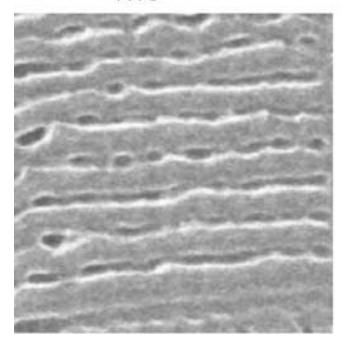

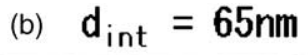

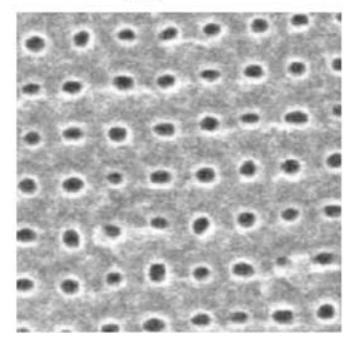

(c) $\mathrm{d}_{\text {int }}=85 \mathrm{~nm}$

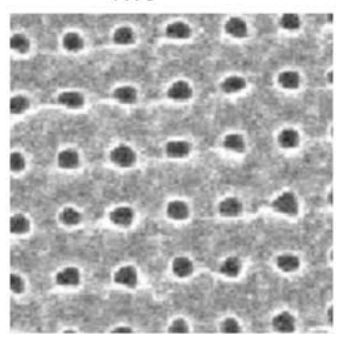

(d) $\mathrm{d}_{\text {int }}=110 \mathrm{~nm}$

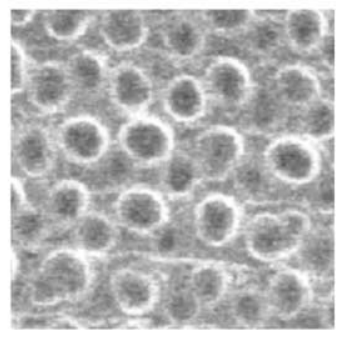

$200 \mathrm{~nm}$

Fig. 4. Porous alumina nanohole array formed by different indentation pitch and the subsequent anodic oxidation. Al surfaces were nanoindented by different intervals from 55 to $110 \mathrm{~nm}$, at the force of $4.16 \times 10^{-5} \mathrm{~N}$, and later they were anodically oxidized at $40 \mathrm{~V}$, 1 min with $0.15 \mathrm{M}$ oxalic acid.

that were formed by anodic oxidation after nanoindentation. Fig. 4 shows SEM micrographs of the surfaces of porous alumina films formed by the anodic oxidation of $40 \mathrm{~V}$ at various indentation intervals. At the interval of $55 \mathrm{~nm}$ (Fig. 4a), nanoholes were connected to each other. On the other hand, at the interval of $110 \mathrm{~nm}$ (Fig. 4d), additional holes were formed randomly between the holes which positions were determined by indentation. The ordered arrays of nanoholes were formed at the intervals of 65 and $80 \mathrm{~nm}$, as shown in Fig. $4 \mathrm{~b}$ and $\mathrm{c}$. The average interval of holes obtained after anodic oxidation was almost the same as the indentation interval, and there was a linear relationship between them when the indentation interval was smaller than $80 \mathrm{~nm}$. However, in the case of $110 \mathrm{~nm}$ interval nanoindentation, the average interval of nanoholes was much smaller than the indented interval. This is due to the formation of additional holes besides those determined by nanoindentation. The spatial order of nanoholes was good when the interval was $65-80$ $\mathrm{nm}$, while it got worse when the interval was either smaller or larger than this range. If there is no indentation, an alumina nanohole trigonal array is formed self-organizingly. The nearest neighbor distance of the self-organized nanoholes at $40 \mathrm{~V}$ is about $90 \mathrm{~nm}$ [8]. If we assume that the cell size is equivalent between triangular and tetragonal arrays, the nearest neighbor nanohole distance in the tetragonal array is estimated to be $78 \mathrm{~nm}$, which is almost the middle of 65 and $85 \mathrm{~nm}$. So we can conclude that if the cell size of the nanoholes differs too much from that of the self-organization condition, the order of the nanohole arrangement becomes poor.

We attempted to form tetragonal as well as trigonal Al dot arrays by selective wet chemical etching of porous alumina films. SEM plan-view images and AFM images of the trigonal as well as tetragonal $\mathrm{Al}$ dots at the $65 \mathrm{~nm}$ indentation interval are shown in Fig. 5. The voltage for anodic oxidation was $30 \mathrm{~V}$. The height and the nearest neighbor distance of the Al dots are 15 and $45 \mathrm{~nm}$ for trigonal array, and 20 and $65 \mathrm{~nm}$ for tetragonal array, respectively.

We further evaluated electron transport property by attaching electrodes around $\mathrm{Al}$ nanodot array. Tetragonal Al dot array was formed by anodic oxidation at $40 \mathrm{~V}$ and indentation interval with $65 \mathrm{~nm}$. Two tungsten electrodes were formed using focused ion beam induced deposition as shown schematically in the inset of Fig. 6. The distance of the gap between electrodes was about $1.5 \mu \mathrm{m}$ and the width of each electrodes was 1.0 $\mu \mathrm{m}$, thus $23 \times 15$ dots array existed between the electrodes. A current-voltage characteristic of A1 dot array are shown in Fig. 6. There is a non-linear current-voltage characteristics at room temperature (Fig. 6a). This is considered to come from tunneling current between the dots, in which alumina barrier exited in between dots. When temperature was lowered to $4.2 \mathrm{~K}$, a suppression of the current near zero bias that was supposed to 
(a)

SEM image of alumina nanoholes

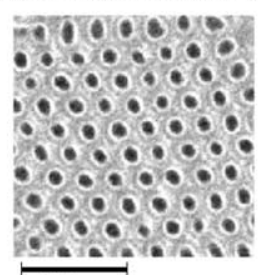

$200 \mathrm{~nm}$
AFM image of Al dots
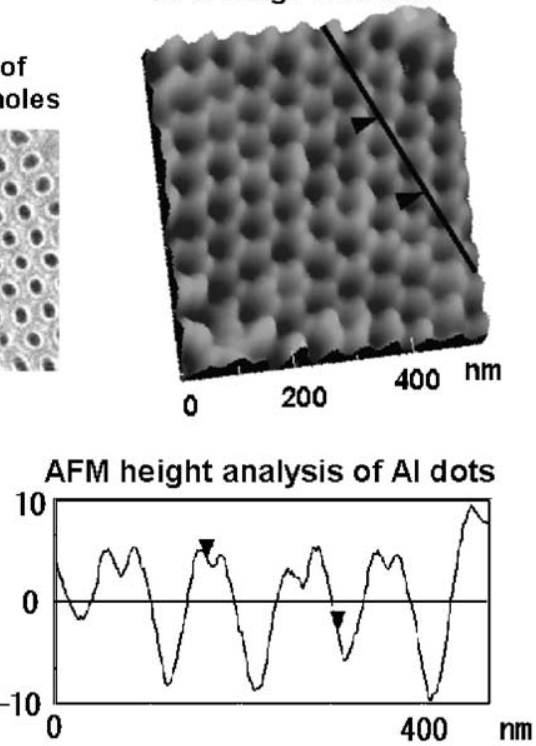

(b)
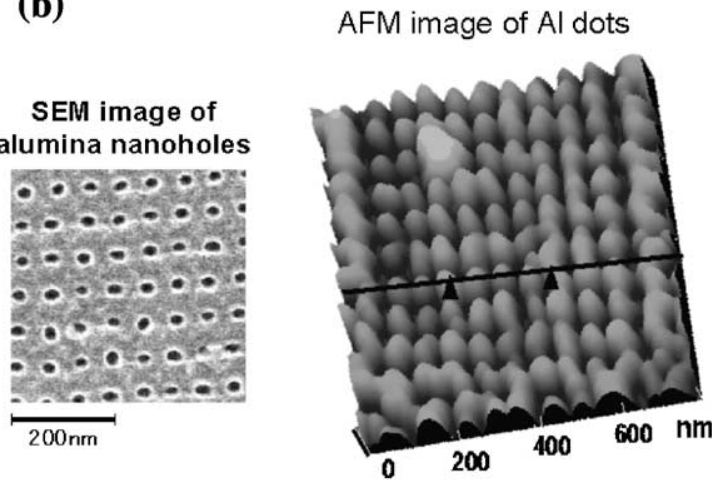

AFM height analysis of Al dots

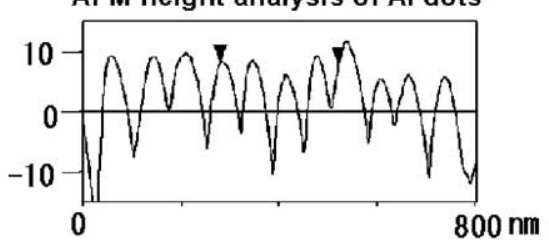

Fig. 5. Trigonal (a) and tetragonal array (b) of nanoholes and Al dots formed by AFM nanoindentation and anodic oxidation. Interval of nanoindentation was $65 \mathrm{~nm}$, and anodic voltage was $30 \mathrm{~V}$. SEM plan view micrographs of the nanohole, AFM image of Al dots after selective wet chemical etching, and its height analysis are shown.

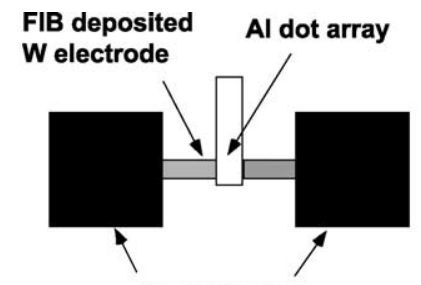

Al electrodes

(a)

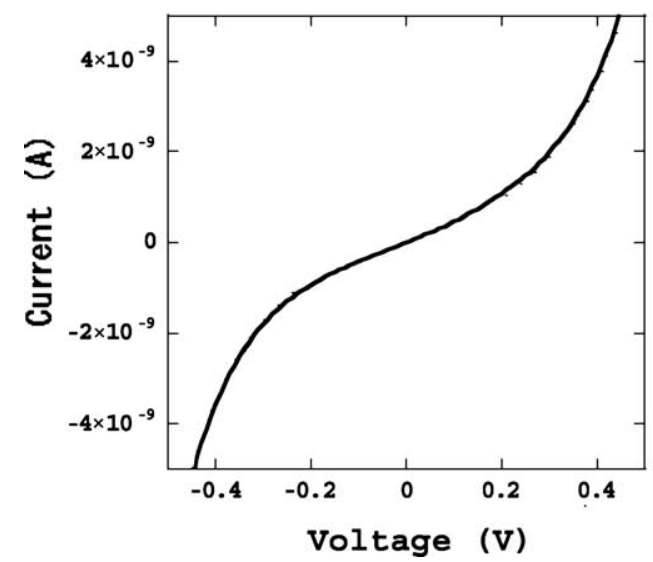

(b)

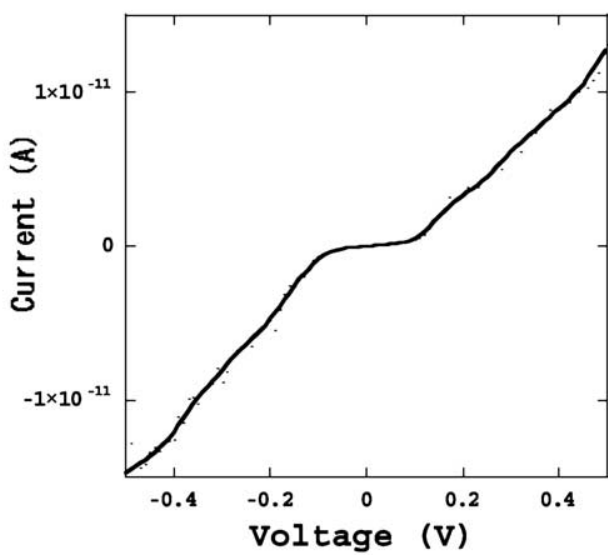

Fig. 6. Current-voltage characteristics of $23 \times 15 \mathrm{Al}$ dot array measured at (a) 298 and (b) $4.2 \mathrm{~K}$. 
come from the Coulomb blockade due to single electron charging effect was observed. The off-set voltage $\left(V_{\text {off }}\right)$ was $0.13 \mathrm{~V}$. If a current passes along the most conductive path with $N$-junctions of Al dots, $V_{\text {off }}$ is approximately equal to $N(e / 2 C)$ [19], and $C$ is estimated to be $2.4 \times 10^{-17} \mathrm{~F}$ assuming that $N$ is 23 . The control of tunneling gap between $\mathrm{Al}$ dots is not so easy since anodic oxidation still continues even after $\mathrm{Al}$ film was broken up to islands. In other samples that were formed almost in the same way, some were completely insulating due to too large gap between $\mathrm{Al}$ dots, and for others cases, Al dots were connected and linear current-voltage characteristics were observed, and further, we observed some kind of hopping conductions in some other sample. Further delicate control of the gap between dots would be needed to observe tunneling effect more stably.

\section{Conclusions}

Porous $\mathrm{Al}$ film was formed on $\mathrm{SiO}_{2} / \mathrm{Si}$ substrate, and after completion of anodic oxidation of $\mathrm{Al}$ film, hexagonal Al dot array was confirmed by cross-sectional SEM and TEM observations. By the use of AFM nanoindentation prior to anodic oxidation, tetragonal array of $\mathrm{Al}$ nanodot that could not be realized by self-organization was formed. The ordered array of alumina nanohole or Al nanodot could be obtained at the indentation interval, which was close to that of the self-organization condition at a given anodic voltage. The results suggest that adjusting the anodic voltage against to the indentation interval can form $\mathrm{Al}$ dot array with a desired geometry. Furthermore, we observed a non-linear current-voltage characteristics in the $\mathrm{Al}$ dots array at $4.2 \mathrm{~K}$, which strongly suggests Coulomb blockade between dots. These results strongly suggest a high potentiality of po- rous alumina based nanofabrication to various nanoelectron devices.

\section{References}

[1] F. Keller, M.S. Hunter, D. Robinson, J. Electrochem. Soc. 100 (1953) 411.

[2] J.P. O'Sullivan, G.C. Wood, Proc. Roy. Soc. London A 317 (1970) 511.

[3] C.G. Granqvist, A. Anderson, O. Hundrei, Appl. Phys. Lett. 35 (1979) 268.

[4] H.L. Hornyak, C.J. Patrissi, C.R. Martin, J. Phys. Chem. B 101 (1997) 1548

[5] D. Routkevitch, T. Bigioni, M. Moskovits, J.M. Xu, J. Phys. Chem. 100 (1996) 14037.

[6] D. Routkevitch, A.A. Tager, J. Haruyama, D. Almawlawi, M. Moskovits, J.M. Xu, IEEE Trans. Electron Devices 43 (1996) 1646.

[7] H. Masuda, K. Fukuda, Science 268 (1995) 146.

[8] S. Shingubara, O. Okino, Y. Sayama, H. Sakaue, T. Takahagi, Jpn. J. Appl. Phys. 36 (1997) 7791.

[9] H. Masuda, F. Kasegawa, S. Ono, J. Electrochem. Soc. 144 (1997) L127.

[10] H. Masuda, K. Yada, A. Osaka, Jpn. J. Appl. Phys. 37 (1998) L1340.

[11] S. Shingubara, O. Okino, H. Sakaue, T. Takahagi, Solid State Electron. 43 (1999) 1143.

[12] S. Shingubara, O. Okino, H. Sakaue, T. Takahagi, J. Vac. Sci. Technol. B 19 (2001) 1901.

[13] Y. Murakami, S. Shingubara, H. Sakaue, T. Takahagi, Digest papers of Microproc. Nanotechnol. 2000 (2000) 178.

[14] S. Shingubara, Y. Murakami, H. Sakaue, T. Takahagi, Jpn. J. Appl. Phys. 41 (2002) L340.

[15] S. Shingubara, Y. Murakami, H. Sakaue, T. Takahagi, Mat. Res. Soc. Symp. Proc. 705 (2002) 133.

[16] I. Amlani, A.O. Orlov, R.K. Kummamuru, Appl. Phys. Lett. 77 (2000) 738.

[17] H. Masuda, H. Yamada, M. Satoh, H. Asoh, Appl. Phys. Lett. 71 (1997) 2770.

[18] H. Asoh, K. Nishio, M. Nakao, T. Tamamura, H. Masuda, J. Electrochem. Soc. 128 (2001) B152.

[19] H. Grabert, M.H. Devoret (Eds.), Single Charge Tunneling - Coulomb Blockade Phenomena in Nanostructures, Nato ASI Series, Prenum Publishing, New York, 1992 (Chapter 7). 